\title{
An Application of Metabolic Syndrome Severity Scores in the Lifestyle Risk Assessment of Taiwanese Adults
}

\author{
Chih-Ming Lin $\mathbb{D}$ \\ Department of Healthcare Information and Management, Ming Chuan University, Taoyuan 333, Taiwan; \\ cmlin@mail.mcu.edu.tw; Tel.: +886-3-350-7001 (ext. 3530)
}

Received: 16 March 2020; Accepted: 8 May 2020; Published: 12 May 2020

\begin{abstract}
A metabolic syndrome (MS) diagnosis was made when the criteria for three or more of five MS components were met. Due to some limitations in the traditional MS criteria, however, different health care societies have sought to develop applicable MS scoring systems instead. Continuous MS scores can be of meaningful value in the prevention, diagnosis, and treatment of MS at different life stages. Relatedly, this study used a database for 27,748 subjects aged 20 to 64 years who received health checks at a health screening institution in Taiwan from 2010 to 2015 to a similar end. Five components of MS (waist circumference, fasting plasma glucose, blood pressure, fasting triglycerides, and high-density lipoprotein) were used to formulate an MS severity score in different gender and age stratums, which was then used to evaluate the risks of various lifestyle habits. Those estimates were then compared with the results for traditional MS diagnosis. The MS severity scores for some behaviors relating to smoking, drinking, physical activity, and sweetened beverage consumption were found to have changed from 0.03 to 0.2 ; however, a logistic regression analysis with dichotomous diagnosis did not indicate significant links between these behaviors and MS. The models established by the MS severity scores can identify the risk factors for MS in a more sensitive manner than the traditional MS diagnosis can, especially with respect to specific lifestyle habits. MS severity score can serve as an indicator to explore the potential risk factors for subclinical conditions in the early stages of MS.
\end{abstract}

Keywords: cardiovascular disease; confirmatory factor analysis; ethnicities; lifestyle; metabolic syndrome

\section{Introduction}

Metabolic syndrome (MS) is a risk factor for type 2 diabetes and cardiovascular diseases (CVD), and it is also associated with various burdens placed on health care systems. As obesity rates rise and population aging continues, the prevalence of MS is also rising. A recent study showed that the prevalence rates of MS in China (21.3\%) and South Korea (31.3\%) have grown by about $30 \%$ over the past 10 years, and nearly doubled in Taiwan over the past 12 years, from $13.6 \%$ to $25.5 \%$ [1]. A China-based study reported that this trend may have been caused by elevated uric acid levels [2]. Nevertheless, MS can be prevented by assessing and addressing of risk factors, such as personal socio-economic conditions and lifestyle behaviors, which include smoking, alcohol consumption, dietary habits, physical activity [3,4]; the intake of antioxidants (e.g., vitamin C and vitamin E) [4-7]; personal income; occupation; and education [8-10].

There has been a question regarding the clinical significance of MS. More specifically, since the five MS components (i.e., waist circumference, fasting plasma glucose (FPG), blood pressure, fasting triglycerides (TG), and high-density lipoprotein (HDL)) are considered CVD risk factors, views still differ on whether another integrated criterion is actually helpful. Gale has argued that MS is 
a redundant diagnosis for people with diabetes, and recommends that the criteria for determining MS should exclude patients with diabetes and CVD [11]. Meanwhile, past studies have found that the continuous/numerical score is more sensitive and less likely to be erroneous when used in statistical analysis than the dichotomous classification [12,13]. Kahn et al. pointed out that, due to information gaps in the current definition of MS, clinicians should assess and treat all relevant risk factors for CVD, rather than only considering the diagnostic criteria for MS. They further suggested that a continuous MS measure could be more efficient for disease prevention [14]. Previous studies on the scoring of continuous MS measures have used several tools such as principle component analysis $[15,16]$, $\mathrm{Z}$ scores [17,18], percentile rankings [19], and factor analysis [20]. Age can be a crucial factor for predicting the development of CVD and needs to be included into any valid scoring system. Relatedly, Eisenmann argued that it is necessary to use the standardized MS score to estimate MS risk due to diabetes, atherosclerosis, and CVD from childhood to adulthood [21]. Continuous MS scores can thus be of meaningful value in the prevention, diagnosis, and treatment of MS at different life stages.

Continuous MS scores have recently been developed for Asian adults. Jung et al. found a high correlation between continuous MS scores and both ischemic stroke and heart disease mortality [22]. Two recent studies developed MS severity scores for Asian populations (i.e., Koreans, Chinese, Malaysians, and Indians) to assess the risk of the development of diabetes and inflammation markers [23,24]. However, few studies have linked continuous MS scores to socioeconomic and lifestyle conditions, as well as to Taiwanese populations. The present study was thus aimed at developing gender- and age-specific MS severity scores for Taiwanese adults, and at assessing the associations between individual lifestyle habits and those severity scores in comparison with the traditional MS diagnosis.

\section{Methods}

\subsection{Data Source}

The study collected and analyzed data from the Major Health Screening Center in Taiwan. The center is a membership-oriented private institution with four clinics located around the country that provide periodic health examinations to the center's members. Each member participated in a check-up program that offers a discounted examination fee for receiving the examination repeatedly over multiple years. The data collection and analysis of the resulting Major Longitudinal Health-Check-Up-Based Population Database (MJLPD) has been described in previous reports [25,26]. The MJLPD database is made accessible to academic researchers upon request. As various ethical issues could arise from data usage, the protocol of this study was evaluated and agreed to by the Research Ethics Committee of National Taiwan University (NTU-REC 201911ES012) and the Major Health Screening Center.

\subsection{Study Sample}

In the study, we collected data from 71,108 participants aged 20 to 64 years who underwent their first standard health screening at the center from 2010-2015. 11,093 participants with CVD including heart diseases, stroke, and diabetes or those receiving related treatments were excluded from the study, since these conditions could produce perturbations in MS-related measures that might mask potentially important relationships. Furthermore, to minimize selection bias, study subjects were calibrated and selected randomly from the remaining participants to reflect the sex and age composition of Taiwan's population. A final total of 27,748 subjects (13,823 males and 13,925 females) met the inclusion criteria for analysis.

\subsection{Response Variables}

In our study, MS was regarded as a dichotomous variable and defined according to the modified Adult Treatment Panel III (ATP III) criteria and the official criteria announced by Taiwan's National 
Health Promotion Administration [27]. A MS diagnosis was made when 3 or more of the following conditions were present: Waist circumference $\geq 90 \mathrm{~cm}$ in men and $\geq 80 \mathrm{~cm}$ in women; fasting plasma glucose (FPG) $\geq 100 \mathrm{mg} / \mathrm{dL}$ ( $5.55 \mathrm{mmol} / \mathrm{L}$ ) or use of antidiabetic medication; systolic blood pressure (SBP) $\geq 130 \mathrm{mmHg}$, diastolic blood pressure $\geq 85 \mathrm{mmHg}$, or use of antihypertensive medication; fasting triglycerides (TG) $\geq 150 \mathrm{mg} / \mathrm{dL}$; and high-density lipoprotein (HDL) cholesterol $<40 \mathrm{mg} / \mathrm{dL}$ in men and $<50 \mathrm{mg} / \mathrm{dL}$ in women.

The general methods for formulating MS severity scores have been reported previously $[20,28]$. Confirmatory factor analysis was performed for the data of adults aged 20-64 years who were categorized into six subgroups based on gender and the following age groups: 20-34, 35-49, and 50-64 years. Those MS severity scores were then presented as scores (i.e., scores that range from theoretical negative to positive infinity and have a mean of 0 and standard deviation (SD) of 1 , as well as a normal distribution) of relative MS severity for a given gender and age range.

\subsection{Explanatory Variables}

The study subjects had each completed a self-administered questionnaire during screening that provided information on their sociodemographic characteristics and lifestyle habits. In addition to sex and age, we collected data regarding four aspects of socioeconomic status (i.e., marital status, education, income, and occupation) and twelve lifestyle habits including smoking, alcohol consumption, betel nut chewing, physical activity (i.e., duration, intensity, and frequency), sleep habits, vegetarian diet, drinking sweetened beverages, and taking nutritional supplements (i.e., vitamin C/E and fish oil), which are all well-documented as constituting related risk factors. Sex, age, and socioeconomic status played the role of confounders in the multi-variable analysis.

\subsection{Statistical Analysis}

Descriptive statistics regarding the above characteristics were calculated for all participants. The prevalence of MS and average MS severity scores were calculated across the gender and age groups. A confirmatory factor analysis approach was used to derive the MS severity scores based on the five MS components, with a weighted contribution for each of the components to a latent MS factor being determined on the basis of both specific age ranges and genders [29]. SBP, rather than diastolic blood pressure, was chosen for this factor analysis because SBP has a stronger association with insulin resistance [30,31]. Furthermore, log-transformed values of TG levels were used because the TG levels of the collected data exhibited a skewed distribution. Meanwhile, in order to be interpretable in a similar manner as the other measures included in the model, inverse values of the HDL cholesterol were utilized. The individual values of five components were then standardized and converted to $Z$ scores before the factor analysis was performed.

We wanted to determine the factor loadings because each one indicated the magnitude of the corresponding association between an associated component and the underlying MS factor. Several fit indices such as the Chi square, Akaike information criterion, root mean square error of approximation, standardized root mean square residual, goodness of fit index, and Bentler-Bonett normed fit index were used to estimate the parameters of the confirmatory factor analyses in accordance with the related criteria [28]. For each of the six subgroups, factor loadings for the five MS components were determined based on one factor (i.e., MS latent factor). Models were constructed, and then selected according to the fit measure indices. Our results showed the factor loadings estimated by the optimal model. The process of factor analysis has been described with formulas in a study conducted by Low et al. [24]. We also calculated MS severity scores based on unstandardized values for the traditional five components in order to provide scores that could be used relatively easily in clinical contexts. These scores were derived by back-transforming the standardized coefficients and a covariance matrix obtained from the factor analysis. The relationships between the dichotomous MS results (that is, whether an individual has or does not have MS), the quartile-ordinal outcomes of the MS severity scores, and the MS severity scores and risk factors were likewise examined by performing a binary 
logistic regression, ordinal regression (i.e., cumulative logistic regression), and linear regression model, respectively. The confirmatory factor analysis and regression analyses were performed using R, AMOS, and SPSS (version 22.0; IBM Institute Inc., New York, NY, USA).

\section{Results}

The overall prevalence rates of MS in this sample were about $16.7 \%$ in men and $7.0 \%$ in women according to the ATP III criteria (Table 1). Women had a lower prevalence of MS, and the prevalence of MS was clearly increased in those more than 50 years old. Upon performing the confirmatory factor analysis of the MS components, we generated models that were well fit to the data (Table 2), with the overall models exhibiting acceptable model fit. Regarding factor loadings, there were notable ageand gender-related differences in the magnitudes of the factor loadings. Among the MS components, waist circumference had the highest factor loadings, indicating that it held the strongest correlation with MS among the investigated sample. SBP and FPG had the lowest factor loadings in men aged 35 to 64 years and women aged 50 to 64 years. Equations based on the factor coefficients from the confirmatory factor analysis results are presented in Table 3. Individual MS severity score can be calculated with the established equation. The mean of the MS severity scores of those with MS and those without MS was 1.259 and -0.167 , respectively. Observing the distributions of the MS severity scores shown in Figure 1, majority of the participants with MS had a severity score of more than zero, and a medium value of 1 and 1.5 for men and women. However, the distributions of the scores for those with MS had major overlaps with the scores for those without MS. The classification cannot be observed clearly until the severity scores are greater than two.

Table 1. Metabolic syndrome and its components for sex- and age-specific groups

\begin{tabular}{|c|c|c|c|c|c|c|c|c|c|c|c|c|}
\hline \multirow{2}{*}{ Groups } & \multirow{2}{*}{$T$} & \multirow{2}{*}{ MS (\%) } & \multicolumn{2}{|c|}{ WC } & \multicolumn{2}{|c|}{ FPG } & \multicolumn{2}{|c|}{ TG } & \multicolumn{2}{|c|}{ HDL-C } & \multicolumn{2}{|c|}{ SBP } \\
\hline & & & Mean & SD & Mean & SD & Mean & SD & Mean & SD & Mean & SD \\
\hline & 13823 & & 82.54 & 8.39 & 101.35 & 14.09 & 124.46 & 76.2 & 53.29 & 12.05 & 119.91 & 13.79 \\
\hline 20-34 YRS & & 11 & 81.37 & 9.17 & 97.8 & 10.29 & 109.26 & 69.93 & 53.99 & 12.02 & 119.39 & 12.52 \\
\hline 35-49 YRS & 4803 & 18.7 & & 8.02 & 101.51 & 13.61 & 136.19 & 79.78 & 51.87 & 11.33 & 119.15 & 13.34 \\
\hline 50-64 YRS & 4204 & 20.6 & 83.23 & 7.7 & 105.23 & 17.05 & 128.46 & 76.03 & 54.11 & 12.72 & 121.36 & 15.46 \\
\hline Women & 13925 & 7 & 70.85 & 7.39 & 96.14 & 12.32 & 87.04 & 52.89 & 66.42 & 15.21 & 109.49 & 15.07 \\
\hline 20-34 YRS & 4583 & 2.1 & 68.18 & 7.14 & 92.42 & 8.28 & 71.57 & 37.28 & 67.42 & 15.09 & 104.59 & 11.53 \\
\hline & 5073 & & & 6.69 & & 9.76 & & 53.63 & 65.41 & 14.78 & 107.91 & 13.62 \\
\hline 50-64 YRS & 4269 & 14.2 & 74.02 & 7.22 & 100.84 & 16.46 & 105.85 & 60.02 & 66.55 & 15.76 & 116.63 & 17.26 \\
\hline
\end{tabular}

Abbreviations: YRS = years, MS = metabolic syndrome; $\mathrm{SD}=$ standard deviation; $\mathrm{WC}=$ waist circumference (in centimeters), FPG = fasting plasma glucose (in mg/dL), TG = triglycerides (in mg/dL), HDL-C = high-density lipoprotein cholesterol (in $\mathrm{mg} / \mathrm{dL}$ ); $\mathrm{SBP}=$ systolic blood pressure (in $\mathrm{mmHg}$ ).

Table 2. Model fit indices and factor loadings used in the confirmatory factor analysis of sex- and age-specific groups

\begin{tabular}{ccccccc}
\hline \multirow{2}{*}{ Indices/Loadings } & \multicolumn{3}{c}{ Men } & \multicolumn{3}{c}{ Women } \\
\cline { 2 - 7 } & 20-34 YRS & 35-49 YRS & 50-64 YRS & 20-34 YRS & 35-49 YRS & 50-64 YRS \\
\hline Indices & & & & & & \\
Chi-square & 85.353 & 96.288 & 88.174 & 23.355 & 65.790 & 31.043 \\
AIC & 107.353 & 118.288 & 110.174 & 45.355 & 87.790 & 53.043 \\
RMSEA & 0.065 & 0.069 & 0.071 & 0.032 & 0.055 & 0.040 \\
SRMR & 0.027 & 0.029 & 0.033 & 0.015 & 0.023 & 0.016 \\
GF1 & 0.993 & 0.992 & 0.992 & 0.998 & 0.995 & 0.997 \\
NFI & 0.947 & 0.962 & 0.958 & 0.988 & 0.978 & 0.988 \\
\hline
\end{tabular}


Table 2. Cont.

\begin{tabular}{|c|c|c|c|c|c|c|}
\hline \multirow{2}{*}{ Indices/Loadings } & \multicolumn{3}{|c|}{ Men } & \multicolumn{3}{|c|}{ Women } \\
\hline & 20-34 YRS & 35-49 YRS & 50-64 YRS & 20-34 YRS & 35-49 YRS & 50-64 YRS \\
\hline \multicolumn{7}{|l|}{ Factor loading } \\
\hline WC & 0.82 & 0.76 & 0.81 & 0.74 & 0.73 & 0.70 \\
\hline FPG & 0.35 & 0.28 & 0.31 & 0.39 & 0.46 & 0.38 \\
\hline Ln-TG & 0.52 & 0.49 & 0.42 & 0.44 & 0.50 & 0.50 \\
\hline HDL-C & 0.40 & 0.36 & 0.38 & 0.35 & 0.38 & 0.41 \\
\hline SBP & 0.41 & 0.35 & 0.24 & 0.38 & 0.37 & 0.36 \\
\hline
\end{tabular}

Abbreviations: YSR = years, AIC = Akaike information criterion, RMSEA = root mean square error of approximation, SRMR = standardized root mean square residual, GFI = goodness of fit index, NFI = Bentler-Bonett normed fit index. Note: GFI, NFI $<0.90$ or RMSEA, SRMR $>0.08$ indicates a poor fit. Factor loadings $>0.3$ were considered clinically meaningful.
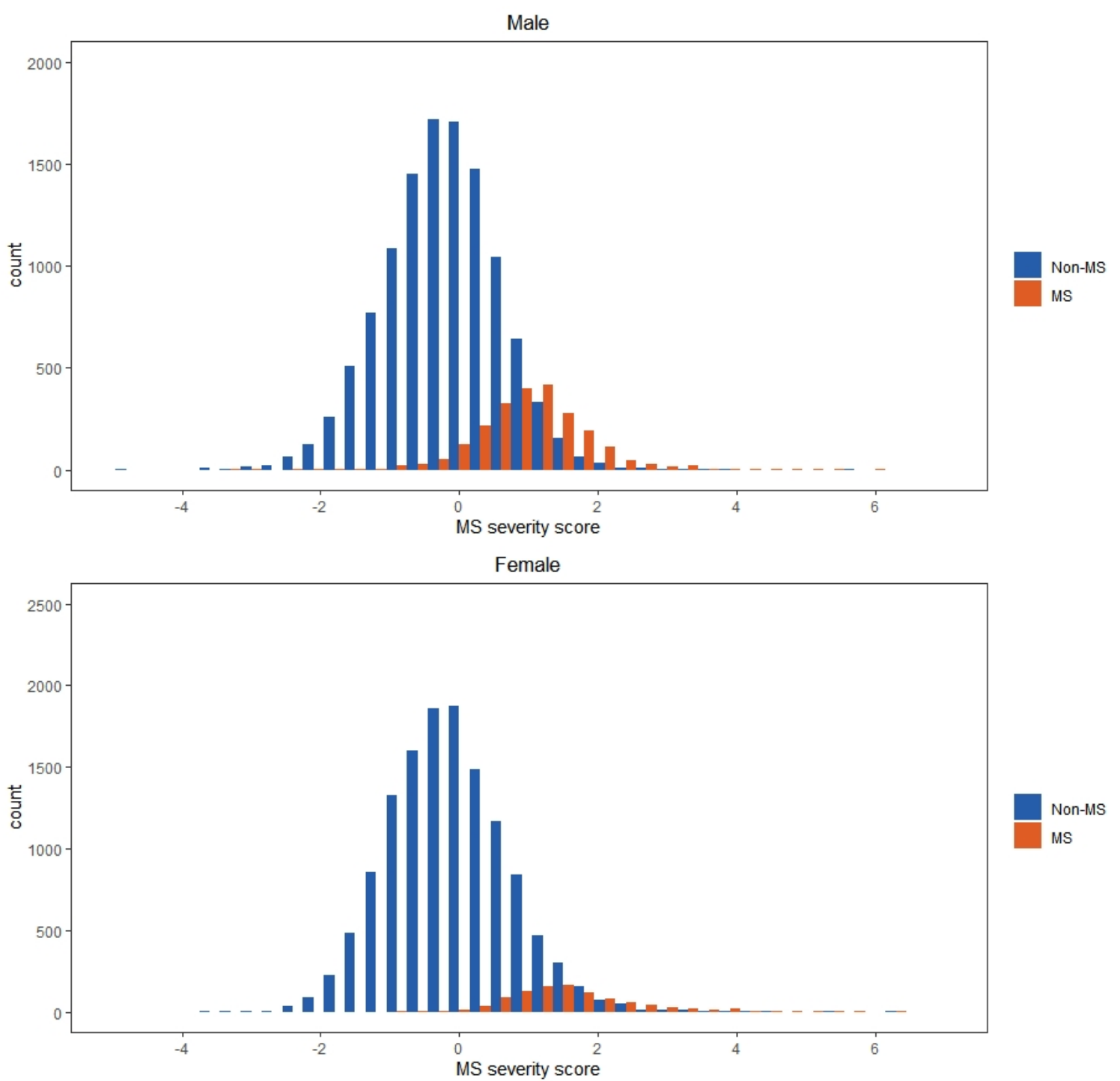

Figure 1. Distributions of MS severity scores in men and women. 
Table 3. Equations used to calculate sex- and age-specific metabolic syndrome severity scores

\begin{tabular}{cc}
\hline Sex-age Groups & Equation \\
\hline Men & \\
20-34 YRS & $-10.6959+0.0844 \times \mathrm{WC}+0.0119 \times \mathrm{FPG}+0.3680 \times \mathrm{Ln}-\mathrm{TG}-0.0082 \times \mathrm{HDL}-\mathrm{C}+0.0121 \times \mathrm{SBP}$ \\
35-49 YRS & $-12.1104+0.0960 \times \mathrm{WC}+0.0094 \times \mathrm{FPG}+0.4556 \times \mathrm{Ln}-\mathrm{TG}-0.0097 \times \mathrm{HDL}-\mathrm{C}+0.0127 \times \mathrm{SBP}$ \\
50-64 YRS & $-11.3783+0.1089 \times \mathrm{WC}+0.0073 \times \mathrm{FPG}+0.2835 \times \mathrm{Ln}-\mathrm{TG}-0.0086 \times \mathrm{HDL}-\mathrm{C}+0.0055 \times \mathrm{SBP}$ \\
Women & \\
20-34 YRS & $-12.6514+0.1032 \times \mathrm{WC}+0.0253 \times \mathrm{FPG}+0.5074 \times \mathrm{Ln}-\mathrm{TG}-0.0100 \times \mathrm{HDL}-\mathrm{C}+0.0175 \times \mathrm{SBP}$ \\
35-49 YRS & $-12.3220+0.0972 \times \mathrm{WC}+0.0246 \times \mathrm{FPG}+0.5251 \times \mathrm{Ln}-\mathrm{TG}-0.0089 \times \mathrm{HDL}-\mathrm{C}+0.0131 \times \mathrm{SBP}$ \\
50-64 YRS & $-11.1397+0.0902 \times \mathrm{WC}+0.0127 \times \mathrm{FPG}+0.5491 \times \mathrm{Ln}-\mathrm{TG}-0.0093 \times \mathrm{HDL}-\mathrm{C}+0.0112 \times \mathrm{SBP}$ \\
\hline
\end{tabular}

MS severity scores are generated by inserting an individual's clinically measured values for WC (in centimeters), FPG (in mg/dL), Ln-TG (in mg/dL), HDL-C (in mg/dL), and SBP (in mmHg); YRS = years.

Table 4 shows the prevalence and severity scores of MS for different lifestyle habits. Unhealthy habits such as smoking, drinking alcohol, chewing betel nut, and drinking sweetened beverages represent higher MS risks, while getting sufficient sleep, engaging in physical activity, and taking vitamin $C$ supplements have positive effects on health. The MS severity scores indicated a more obvious link to lifestyle habits, especially for physical activity and drinking sweetened beverages, and this link was not observed in the dichotomous diagnosis.

Table 4. MS prevalence and severity scores categorized according to lifestyle habits

\begin{tabular}{|c|c|c|c|c|c|}
\hline \multirow{2}{*}{ Lifestyle Habit } & \multicolumn{2}{|c|}{ MS } & \multicolumn{2}{|c|}{ MS Severity Score } & \multirow{2}{*}{ Total } \\
\hline & $n$ & $\%$ & Mean (SD) & Median & \\
\hline \multicolumn{6}{|l|}{ Smoking } \\
\hline None & 1939 & $9.9 \%$ & $-0.024(0.974)$ & -0.079 & 19,590 \\
\hline Second-hand smoke & 118 & $11.0 \%$ & $0.058(1.126)$ & -0.044 & 1069 \\
\hline Quit & 281 & $17.0 \%$ & $0.038(0.970)$ & -0.011 & 1652 \\
\hline Casual intake & 135 & $13.8 \%$ & 0.017 (1.003) & -0.032 & 978 \\
\hline Daily intake & 641 & $18.3 \%$ & $0.080(1.015)$ & 0.032 & 3495 \\
\hline Missing data & 168 & $17.4 \%$ & $0.108(1.301)$ & 0.038 & 964 \\
\hline \multicolumn{6}{|l|}{ Drinking } \\
\hline None & 2297 & $10.7 \%$ & $-0.004(0.987)$ & -0.066 & 21,384 \\
\hline Quit & 67 & $13.3 \%$ & $0.027(0.973)$ & 0.008 & 502 \\
\hline 1-2 times/wk & 400 & $15.3 \%$ & $-0.001(0.982)$ & -0.039 & 2608 \\
\hline 3-4 times/wk & 150 & $17.5 \%$ & $-0.057(1.002)$ & -0.068 & 858 \\
\hline$>4$ times/wk & 58 & $19.1 \%$ & $-0.026(0.957)$ & -0.054 & 304 \\
\hline Missing data & 310 & $14.8 \%$ & $0.082(1.154)$ & 0.025 & 2092 \\
\hline \multicolumn{6}{|l|}{ Chewing betel nut } \\
\hline None & 2728 & $10.9 \%$ & $-0.015(0.981)$ & -0.067 & 25,105 \\
\hline Quit & 24 & $40.7 \%$ & $0.166(1.043)$ & 0.105 & 840 \\
\hline $1-2$ times/wk & 70 & $27.9 \%$ & $0.352(1.199)$ & 0.341 & 251 \\
\hline 3-4 times/wk & 194 & $23.1 \%$ & $0.567(1.045)$ & 0.612 & 59 \\
\hline$>4$ times/wk & 24 & $25.8 \%$ & $0.189(0.978)$ & 0.026 & 93 \\
\hline Missing data & 242 & $17.3 \%$ & $0.096(1.215)$ & 0.003 & 1400 \\
\hline \multicolumn{6}{|l|}{ Sleeping (hrs/day) } \\
\hline$<4$ & 45 & $13.8 \%$ & $0.082(1.052)$ & -0.034 & 327 \\
\hline $4.0-5.9$ & 719 & $12.1 \%$ & $0.029(1.010)$ & -0.034 & 5959 \\
\hline $6.0-6.9$ & 1592 & $12.0 \%$ & $-0.012(0.974)$ & -0.059 & 13,267 \\
\hline $7.0-7.9$ & 681 & $10.7 \%$ & $-0.016(0.985)$ & -0.063 & 6349 \\
\hline$\geq 8$ & 104 & $10.1 \%$ & $-0.029(1.004)$ & -0.113 & 1031 \\
\hline Missing data & 141 & $17.3 \%$ & $0.165(1.353)$ & 0.078 & 815 \\
\hline
\end{tabular}


Table 4. Cont.

\begin{tabular}{|c|c|c|c|c|c|}
\hline \multirow{2}{*}{ Lifestyle Habit } & \multicolumn{2}{|c|}{ MS } & \multicolumn{2}{|c|}{ MS Severity Score } & \multirow{2}{*}{ Total } \\
\hline & $n$ & $\%$ & Mean (SD) & Median & \\
\hline \multicolumn{6}{|c|}{ Physical activity (level) } \\
\hline None & 2311 & $11.9 \%$ & $0.030(0.998)$ & -0.024 & 19,344 \\
\hline Light & 588 & $11.1 \%$ & $-0.069(0.937)$ & -0.120 & 5313 \\
\hline Moderate & 120 & $8.4 \%$ & $-0.230(0.897)$ & -0.259 & 1426 \\
\hline Heavy & 42 & $11.6 \%$ & $0.039(1.066)$ & -0.031 & 363 \\
\hline Missing data & 221 & $17.0 \%$ & 0.109 (1.277) & 0.006 & 1302 \\
\hline \multicolumn{6}{|c|}{ Physical activity (times/wk) } \\
\hline None & 881 & $12.0 \%$ & $-0.011(1.038)$ & -0.080 & 7340 \\
\hline 1 & 497 & $11.9 \%$ & $-0.059(0.950)$ & -0.086 & 4189 \\
\hline $2-3$ & 644 & $10.7 \%$ & $-0.057(0.960)$ & -0.104 & 6003 \\
\hline 7 & 812 & $11.2 \%$ & $0.009(1.016)$ & -0.054 & 7265 \\
\hline$>7$ & 129 & $12.9 \%$ & $0.049(0.978)$ & -0.011 & 997 \\
\hline Missing data & 319 & $16.3 \%$ & $0.117(1.187)$ & 0.027 & 1954 \\
\hline \multicolumn{6}{|c|}{ Physical activity (hrs/day) } \\
\hline$<0.5$ & 948 & $11.4 \%$ & $0.027(0.993)$ & -0.031 & 8305 \\
\hline $0.5-1$ & 1162 & $11.4 \%$ & $-0.006(0.991)$ & -0.052 & 10,198 \\
\hline $1-2$ & 576 & $10.9 \%$ & $-0.055(0.964)$ & -0.118 & 5267 \\
\hline$>2$ & 223 & $13.3 \%$ & $-0.021(0.959)$ & -0.053 & 1679 \\
\hline Missing data & 373 & $16.2 \%$ & $0.093(1.156)$ & -0.009 & 2299 \\
\hline \multicolumn{6}{|l|}{ Vegetarian diet } \\
\hline No & 3145 & $11.7 \%$ & $-0.002(0.989)$ & -0.057 & 26,804 \\
\hline Yes & 98 & $12.8 \%$ & $0.043(1.085)$ & -0.026 & 766 \\
\hline Missing data & 39 & $21.9 \%$ & $0.389(1.794)$ & 0.348 & 178 \\
\hline \multicolumn{6}{|c|}{ Drinking sweetened beverages (cups/wk) } \\
\hline None & 1236 & $12.2 \%$ & $-0.023(0.986)$ & -0.066 & 10,167 \\
\hline $1-3$ & 364 & $10.5 \%$ & $0.017(0.991)$ & -0.043 & 3464 \\
\hline $4-6$ & 403 & $10.8 \%$ & $0.020(0.942)$ & -0.031 & 3727 \\
\hline 7 & 999 & $11.7 \%$ & $-0.022(0.969)$ & -0.070 & 8508 \\
\hline$>7$ & 172 & $13.7 \%$ & $0.044(1.150)$ & -0.069 & 1260 \\
\hline Missing data & 108 & $17.4 \%$ & $0.142(1.418)$ & 0.007 & 622 \\
\hline \multicolumn{6}{|c|}{ Taking vitamin $C$ supplements } \\
\hline No & 2927 & $12.2 \%$ & $0.013(1.004)$ & -0.042 & 23,983 \\
\hline Yes & 355 & $9.4 \%$ & $-0.073(0.967)$ & -0.116 & 3765 \\
\hline \multicolumn{6}{|c|}{ Taking vitamin E supplements } \\
\hline No & 3096 & $11.9 \%$ & $0.006(1.003)$ & -0.049 & 26,121 \\
\hline Yes & 186 & $11.4 \%$ & $-0.058(0.951)$ & -0.105 & 1627 \\
\hline \multicolumn{6}{|c|}{ Taking fish oil supplements } \\
\hline No & 3063 & $11.7 \%$ & $0.001(0.999)$ & -0.055 & 26,172 \\
\hline Yes & 219 & $13.9 \%$ & $0.037(1.010)$ & -0.019 & 1576 \\
\hline
\end{tabular}

Abbreviations: $\mathrm{MS}=$ metabolic syndrome; $\mathrm{SD}$ = standard deviation.

Using three regression models, the risk factors for MS or its severity were estimated (Table 5). With the MS severity score as the outcome variable, it was observed that persons who smoked had a $11-13 \%$ increased risk of MS, or a severity score of 0.07 , compared to non-smokers. Drinking alcohol more than 3 times a week caused a $17-25 \%$ decrease in MS risk, or a severity score of $0.12-0.19$. Drinking sweetened beverages week resulted in more than 0.03 severity score. Unlike the findings with the MS severity score, the three lifestyle habits were found to have no relation to MS in a logistic regression analysis with the dichotomous diagnosis. Similar observations were made for physical activity frequency; that is, regular exercise was found to protect against MS when using the model with the severity score, but the benefit could not be observed in persons who exercised when using the model with the dichotomous diagnosis. Betel nut intake was also found to have an adverse effect on MS when a logistic model was used, but the effect could not be found when using a linear model. Irrespective of the model used, meanwhile, our multi-variable analysis showed that sleep, duration of physical activity, vegetarian diet, and taking vitamin E supplements were not related to the occurrence 
of MS or its severity. Furthermore, vitamin C and fish oil intake were found to have opposite effects on MS severity when using a linear model.

Table 5. Risk factors of lifestyle habits for metabolic syndrome in different regression models

\begin{tabular}{|c|c|c|c|c|c|c|}
\hline \multirow{2}{*}{ Lifestyle Habit } & \multicolumn{2}{|c|}{ Logistic Regression } & \multicolumn{2}{|c|}{ Ordinal Regression } & \multicolumn{2}{|c|}{ Linear Regression } \\
\hline & AOR & $p$-Value & AOR & $p$-Value & $\beta$ & $p$-Value \\
\hline \multicolumn{7}{|l|}{ Smoking (vs. None) } \\
\hline Second-hand smoke & 1.100 & 0.452 & 1.002 & 0.782 & 0.060 & 0.113 \\
\hline Quit & 1.104 & 0.684 & 1.132 & 0.027 & 0.071 & 0.022 \\
\hline Intake casually & 1.077 & 0.731 & 1.110 & 0.129 & 0.065 & 0.092 \\
\hline Intake everyday & 1.136 & 0.505 & 1.112 & 0.019 & 0.068 & 0.007 \\
\hline \multicolumn{7}{|l|}{ Drink (vs. None) } \\
\hline Quit & 0.819 & 0.228 & 0.832 & 0.062 & -0.122 & 0.027 \\
\hline $1-2$ times/wk & 0.951 & 0.502 & 0.914 & 0.045 & -0.048 & 0.053 \\
\hline 3-4 times/wk & 0.956 & 0.757 & 0.830 & 0.013 & -0.134 & 0.001 \\
\hline$>4$ times/wk & 0.854 & 0.414 & 0.752 & 0.023 & -0.191 & 0.008 \\
\hline \multicolumn{7}{|c|}{ Chewing betel nut (vs. None) } \\
\hline Quit & 1.272 & 0.033 & 1.106 & 0.213 & -0.163 & 0.250 \\
\hline 1-2 times/wk & 1.859 & 0.001 & 1.561 & 0.002 & 0.083 & 0.061 \\
\hline 3-4 times/wk & 2.973 & 0.004 & 3.216 & 0.001 & 0.122 & 0.437 \\
\hline$>4$ times/wk & 1.060 & 0.869 & 1.375 & 0.208 & 0.323 & 0.165 \\
\hline \multicolumn{7}{|l|}{ Sleep (vs. $\geq 8$ hrs/day) } \\
\hline$<4 \mathrm{hrs} /$ day & 1.034 & 0.888 & 1.141 & 0.348 & 0.025 & 0.761 \\
\hline $4.0-5.9 \mathrm{hrs} /$ day & 0.959 & 0.759 & 1.108 & 0.153 & 0.027 & 0.692 \\
\hline $6.0-6.9 \mathrm{hrs} /$ day & 0.982 & 0.891 & 1.107 & 0.325 & 0.038 & 0.114 \\
\hline 7.0-7.9 hrs/day & 0.896 & 0.416 & 1.105 & 0.491 & -0.014 & 0.672 \\
\hline \multicolumn{7}{|c|}{ Physical activity level (vs. None) } \\
\hline Light & 0.792 & $<0.001$ & 0.837 & $<0.001$ & -0.088 & $<0.001$ \\
\hline Moderate & 0.572 & $<0.001$ & 0.653 & $<0.001$ & -0.236 & $<0.001$ \\
\hline Heavy & 0.669 & 0.251 & 0.742 & 0.066 & -0.221 & 0.016 \\
\hline \multicolumn{7}{|c|}{ Physical activity frequency (vs. None) } \\
\hline 1 time/wk & 0.793 & 0.075 & 0.935 & 0.366 & -0.003 & 0.873 \\
\hline 2-3 times/wk & 0.853 & 0.210 & 0.876 & 0.003 & -0.038 & 0.091 \\
\hline 7 times/wk & 0.899 & 0.395 & 0.940 & 0.125 & -0.085 & 0.001 \\
\hline$>7$ times/wk & 0.977 & 0.853 & 0.988 & 0.744 & -0.055 & 0.178 \\
\hline \multicolumn{7}{|c|}{ Physical activity duration (vs. $<0.5$ hrs/day) } \\
\hline $0.5-1$ hrs/day & 1.044 & 0.491 & 1.027 & 0.439 & 0.024 & 0.453 \\
\hline 1-2 hrs/day & 0.966 & 0.649 & 0.914 & 0.355 & 0.038 & 0.347 \\
\hline$>2$ hrs/day & 0.935 & 0.511 & 0.943 & 0.317 & -0.014 & 0.490 \\
\hline \multicolumn{7}{|l|}{ Vegetarian diet (vs. No) } \\
\hline Yes & 1.003 & 0.983 & 0.989 & 0.889 & -0.007 & 0.800 \\
\hline \multicolumn{7}{|c|}{ Drinking sweetened beverages (vs. None) } \\
\hline $1-3$ cups/wk & 1.115 & 0.057 & 1.024 & 0.450 & 0.038 & 0.032 \\
\hline $4-6$ cups/wk & 1.004 & 0.961 & 1.060 & 0.150 & 0.032 & 0.146 \\
\hline 7 cups/wk & 0.983 & 0.822 & 0.984 & 0.704 & -0.006 & 0.780 \\
\hline$>7$ cups/wk & 1.080 & 0.479 & 0.985 & 0.813 & 0.052 & 0.037 \\
\hline \multicolumn{7}{|c|}{ Taking vitamin $\mathrm{C}$ supplements (vs. No) } \\
\hline Yes & 0.849 & 0.036 & 0.883 & 0.001 & -0.067 & 0.002 \\
\hline \multicolumn{7}{|c|}{ Taking vitamin E supplements (vs. No) } \\
\hline Yes & 1.046 & 0.671 & 0.945 & 0.332 & -0.039 & 0.225 \\
\hline \multicolumn{7}{|c|}{ Taking fish oil supplements (vs. No) } \\
\hline Yes & 1.204 & 0.048 & 1.095 & 0.110 & 0.071 & 0.022 \\
\hline
\end{tabular}

Adjusted odds ratios (AOR) and $\beta$ are calculated by three regression models adjusted with all covariates, including demographic variables and socioeconomic status. Logistic regressions are calculated with ATP III criteria for MS. Linear regression and ordinal logistic regressions are calculated with MS severity score and its quartile outcome.

\section{Discussions}

The present study calculated MS severity scores for Taiwanese adults (other than elderly adults) that take variations in how MS is manifested in different gender and age groups into account by placing differential weights on individual MS components. Waist circumference and TG levels were found to dominate the contributions of the factor loadings for MS. HDL cholesterol levels had particularly high 
factor loadings among older women. SBP exhibited lower factor loadings than the other components, which corresponds to the findings of previous studies $[23,28]$. The majority of the participants with MS had a positive score, which implies that zero may be a critical cut-off point for evaluating MS severity. Moreover, the large overlap in the score distribution of those diagnosed with MS and those not diagnosed with MS might mean that there are lots of individuals who do not suffer from MS but who have high severity scores due to having been neglected by clinics for potential risk of CVD. We also revealed that the MS severity scores in our study were highly correlated with lifestyle habits-including smoking, drinking alcohol, consuming sweetened beverages, and physical activity-which was not the case, however, for traditional MS diagnosis. To our knowledge, this is the first study to investigate an age- and gender-specific MS scoring system for Taiwanese adults based on the contribution of individual MS components, and to apply the system to the underlying risk factors.

Some confusion may arise from the traditional ATP III criteria, such as whether individuals with two high level MS components have lower CVD risk than those whose levels are slightly above the criteria in three or more components. By focusing on the purpose of health education, physicians can provide advice on disease prevention based on the risk severity levels for different groups and, consequently, promote public health $[29,30]$. The World Health Organization has stated that MS may not be suitable for clinical diagnosis although new community-based prevention strategies should nonetheless be developed and evaluated [31]. In addition, as age plays an important effector role in CVD, the traditional MS classification criteria may be questionable given their failure to consider age [30,32]. Eisenmann has argued, meanwhile, that a continuous MS score that is estimated from a specific population cannot be generalized. In other words, the MS score formulas used for different ethnic, sex, and age subgroups should be different [21]. Thus, estimations of MS severity or CVD risk should be established and calibrated based on specific populations. The present study provided a solution that addresses the question regarding the clinical significance of MS, while also evaluating the risk factors of MS. In the future, relationship verification and personal calculator development could be carried out used the established sex-age equation, so as to apply the MS severity scores of individuals in a clinical context and assess temporal changes in cardiometabolic risk.

Previous studies that estimated the $\mathrm{Z}$ scores of MS components for men and women showed that such a method can be helpful in making clinical assessments of MS correlations between parents and children, and could even be used to develop applications that can used on a calculator [33,34]. Guseman et al. noted that most studies have used cross-sectional designs and neglected to consider age; however, there is insufficient evidence to prove whether or not high risk scores in adolescence are related to CVD risk in adulthood, so it is necessary to develop indicators which could be applied to longitudinal estimation during different life stages [33]. Using the MS risk scoring system developed by Sullivan et al. and adjusting it with age factors in a Chinese population, Kang et al. created a recipient's operational characteristic curve estimation of CVD risk, and found that continuous MS scores are more accurate in predicting CVD risk. They also found that the continuous MS score is not linearly related to CVD risk, and suggested that future scores should be developed for different racial, sex, and age subgroups [35]. Our findings revealed that the use of ordinal or linear regression analysis combined with sex- and age-specific MS severity scores is more sensitive in terms of identifying the related risk factors, a revelation which provides some empirical references for future research.

Another well-known continuous indicator for predicting CVD other than MS scores is the Framingham risk score (FRS), which considers age and can predict CVD risk for the following 10 years. A person is considered to have relatively low risk if the FRS is less than $10 \%$. Nevertheless, due to lack of estimation of obesity and blood glucose levels, some studies have suggested that the FRS has limitations in the development of disease prediction and prevention strategies for different age groups [36,37]. A study investigating an Asian-Indian population noted that using the FRS to estimate CVD risk for Asian populations could result in underestimations, which might be present mainly in children and adolescents and MS patients [38]. Using the same data source as the present study, Liao and Lin recently determined FRS values for patients diagnosed with MS but without diabetes 
or other CVD, and found that these relatively healthy MS cases had fairly low FRS results (with an average of only 1.4\%) [25]. Thus, a relatively low FRS could be easily ignored by physicians or patients and thus be ineffective for the purpose of early disease prevention.

The present study has a credible study design. In addition to exploring potential risk factors by analyzing a large sample database, this study also established a continuous MS indicator stratified by gender and age for a specific Asian population. Nevertheless, there are also limitations that need to be considered. As this is a cross-sectional study, its analyses cannot be used to establish causal relationships. Additionally, some independent variables were missing, which may diminish the validity of the study slightly. Moreover, its participants were healthier than the general population because those who had CVD or took related medicines and the elderly were excluded. As such, the risk estimations cannot be suitable for adults other than those who met the selection criteria.

\section{Conclusions}

Not intending to replace the function of traditional MS diagnosis, this study adds evidence for wide application of MS severity scores among an Asian population. Due to being more sensitive than traditional MS diagnosis when used to predict MS risks associated with various lifestyle habits, the use of MS severity scores can be promoted among individuals or health institutions for disease prevention. Health caregivers can utilize MS severity scores to propose or assess lifestyle-related prevention strategies for those at risk of underlying disease by monitoring the fluctuations in their MS severity scores over time.

Funding: This study was supported by a grant from the Ministry of Science and Technology (MOST 107-2410-H-130-051).

Acknowledgments: The author thank Chen-Mao Liao for statistics consultation, and Wan-Ting Hung and Chin-Chia Kuo for assistance with processing of data. All or part of the data used in this research were authorized by, and received from MJ Health Research Foundation (Authorization Code: MJHRF2017003A \& MJHRF2018013A). Any interpretation or conclusion described in this paper does not represent the views of MJ Health Research Foundation.

Conflicts of Interest: The author declare no conflict of interest. The founding sponsors had no role in the design of the study; in the collection, analyses, or interpretation of data; in the writing of the manuscript, or in the decision to publish the results.

\section{References}

1. Ranasinghe, P.; Mathangasinghe, Y.; Jayawardena, R.; Hills, A.P.; Misra, A. Prevalence and trends of metabolic syndrome among adults in the Asia-Pacific region: A systematic review. BMC Public Health 2017, $17,101$. [CrossRef]

2. Zhou, H.; Ma, Z.F.; Lu, Y.; Du, Y.; Shao, J.; Wang, L.; Wu, Q.; Pan, B.; Zhu, W.; Zhao, Q.; et al. Elevated serum uric acid, hyperuricaemia and dietary patterns among adolescents in mainland China. J. Pediatr. Endocrinol. Metab. 2020, 33, 487-493. [CrossRef] [PubMed]

3. Jahangiry, L.; Shojaeizadeh, D.; Montazeri, A.; Najafi, M.; Mohammad, K.; Farhangi, M.A. Modifiable lifestyle risk factors and metabolic syndrome: Opportunities for a web based preventive program. J. Res. Health Sci. 2014, 14, 303-307. [PubMed]

4. Racette, S.B.; Deusinger, S.S.; Inman, C.L.; Burlis, T.L.; Highstein, G.R.; Buskirk, T.D.; Steger-May, K.; Peterson, L.R. Worksite Opportunities for Wellness (WOW): Effects on cardiovascular disease risk factors after 1 year. Prev. Med. 2009, 49, 108-114. [CrossRef]

5. Kim, J.; Choi, Y.H. Physical activity, dietary vitamin C, and metabolic syndrome in Korean adults: The Korea National Health and Nutrition Examination Survey 2008 to 2012. Public Health 2016, 135, 30-37. [CrossRef] [PubMed]

6. Li, Y.; Guo, H.; Wu, M.; Liu, M. Serum and dietary antioxidant status is associated with lower prevalence of the metabolic syndrome in a study in Shanghai, China. Asia Pac. J. Clin. Nutr. 2013, 22, 60-68. [PubMed]

7. Suzuki, K.; Ito, Y.; Inoue, T.; Hamajima, N. Inverse association of serum carotenoids with prevalence of metabolic syndrome among Japanese. Clin. Nutr. 2011, 30, 369-375. [CrossRef] [PubMed] 
8. Marquezine, G.F.; Oliveira, C.M.; Pereira, A.C.; Krieger, J.E.; Mill, J.G. Metabolic syndrome determinants in an urban population from Brazil: Social class and gender-specific interaction. Int. J. Cardiol. 2008, 129, 259-265. [CrossRef]

9. Yoo, S.; Cho, H.J.; Khang, Y.H. General and abdominal obesity in South Korea, 1998-2007: Gender and socioeconomic differences. Prev. Med. 2010, 51, 460-465. [CrossRef]

10. Kim, J.Y.; Kim, S.H.; Cho, Y.J. Socioeconomic status in association with metabolic syndrome and coronary heart disease risk. Korean J. Fam. Med. 2013, 34, 131-138. [CrossRef]

11. Gale, E.A. Should we dump the metabolic syndrome?: Yes. BMJ 2008, 336, 640. [CrossRef]

12. Ragland, D.R. Dichotomizing continuous outcome variables: Dependence of the magnitude of association and statistical power of the cut point. Epidemiology 1992, 3, 434-440. [CrossRef]

13. Steele, R.M.; Brage, S.; Corder, K.; Wareham, N.J.; Ekelund, U. Physical activity, cardiorespiratory fitness, and the metabolic syndrome in youth. J. Appl. Physiol. 2008, 105, 342-351. [CrossRef] [PubMed]

14. Kahn, R.; Buse, J.; Ferrannini, E.; Stern, M. The metabolic syndrome: Time for a critical appraisal. Diabetologia 2005, 48, 1684-1699. [CrossRef]

15. Katzmarzyk, P.T.; Perusse, L.; Malina, R.M.; Bergeron, J.; Despres, J.; Bouchard, C. Stability of indicators of the metabolic syndrome from childhood and adolescence to young adulthood: The Quebec Family Study. J. Clin. Epidemiol. 2001, 54, 190-195. [CrossRef]

16. Wijndaele, K.; Beunen, G.; Duvigneaud, N.; Matton, L.; Duquet, W.; Thomis, M.; Lefevre, J.; Philippaerts, R.M. A continuous metabolic syndrome risk score: Utility for epidemiological analyses. Diabetes Care 2006, 29, 2329. [CrossRef] [PubMed]

17. Eisenmann, J.C.; Welk, G.J.; Wickel, E.E.; Blair, S.N. Combined influence of cardiorespiratory fitness and body mass index on cardiovascular disease risk factors among 8-18 year old youth: The Aerobics Center Longitudinal Study. Int. J. Pediatr. Obes. 2007, 2, 66-72. [CrossRef]

18. Andersen, L.B.; Harro, M.; Sardinha, L.B.; Froberg, K.; Ekelund, U.; Brage, S.; Anderssen, S.A. Physical activity and clustered cardiovascular risk in children: A cross-sectional study (The European Youth Heart Study). Lancet 2006, 368, 299-304. [CrossRef]

19. Raitakari, O.T.; Porkka, K.V.; Rasanen, L.; Ronnemaa, T.; Viikari, J.S. Clustering and six year cluster-tracking of serum total cholesterol, HDL-cholesterol and diastolic blood pressure in children and young adults: The Cardiovascular Risk in Young Finns Study. J. Clin. Epidemiol. 1994, 47, 1085-1093. [CrossRef]

20. Gurka, M.J.; Ice, C.L.; Sun, S.S.; DeBoer, M.D. A confirmatory factor analysis of the metabolic syndrome in adolescents: An examination of sex and racial/ethnic differences. Cardiovasc. Diabetol. 2012, 11, 128. [CrossRef]

21. Eisenmann, J.C. On the use of a continuous metabolic syndrome score in pediatric research. Cardiovasc. Diabetol. 2008, 7, 17. [CrossRef] [PubMed]

22. Jung, K.J.; Jee, Y.H.; Jee, S.H. Metabolic risk score and vascular mortality among Korean adults: The Korean Metabolic Syndrome Mortality Study. Asia. Pac. J. Public Health 2017, 29, 122-131. [CrossRef] [PubMed]

23. Huh, J.H.; Lee, J.H.; Moon, J.S.; Sung, K.C.; Kim, J.Y.; Kang, D.R. Metabolic syndrome severity score in Korean adults: Analysis of the 2010-2015 Korea National Health and Nutrition Examination Survey. J. Korean Med. Sci. 2019, 34, e48. [CrossRef] [PubMed]

24. Low, S.; Khoo, K.C.J.; Wang, J.; Irwan, B.; Sum, C.F.; Subramaniam, T.; Lim, S.C.; Wong, T.K.M. Development of a metabolic syndrome severity score and its association with incident diabetes in an Asian population-Results from a longitudinal cohort in Singapore. Endocrine 2019, 65, 73-80. [CrossRef]

25. Yang, X.; Tao, Q.; Sun, F.; Zhan, S. The impact of socioeconomic status on the incidence of metabolic syndrome in a Taiwanese health screening population. Int. J. Public Health 2012, 57, 551-559. [CrossRef]

26. Liao, C.M.; Lin, C.M. Life course effects of socioeconomic and lifestyle factors on metabolic syndrome and 10-year risk of cardiovascular disease: A longitudinal study in Taiwan adults. Int. J. Environ. Res. Public Health 2018, 15, 2178. [CrossRef]

27. Taiwan National Health Promotion Administration. 2007 Criteria of Metabolic Syndrome in Adults. Available online: https://www.hpa.gov.tw/Pages/Detail.aspx?nodeid=639\&pid=1219 (accessed on 5 March 2019).

28. Gurka, M.J.; Lilly, C.L.; Oliver, M.N.; DeBoer, M.D. An examination of sex and racial/ethnic differences in the metabolic syndrome among adults: A confirmatory factor analysis and a resulting continuous severity score. Metabolism 2014, 63, 218-225. [CrossRef] 
29. Sattar, N.; Forouhi, N.G. Metabolic syndrome criteria: Ready for clinical prime time or work in progress? Eur. Heart J. 2005, 26, 1249-1251. [CrossRef]

30. Kastelein, J. Cardiovascular risk-Through the ages. Atheroscler. Suppl. 2004, 5, 1-2. [CrossRef]

31. Simmons, R.K.; Alberti, K.G.M.M.; Gale, E.A.M.; Colagiuri, S.; Tuomilehto, J.; Qiao, Q.; Ramachandran, A.; Tajima, N.; Mirchov, I.B.; Ben-Nakhi, A.; et al. The metabolic syndrome: Useful concept or clinical tool? Report of a WHO Expert Consultation. Diabetologia 2010, 53, 600-605. [CrossRef]

32. Tuomilehto, J. Impact of age on cardiovascular risk: Implications for cardiovascular disease management. Atheroscler. Suppl. 2004, 5, 9-17. [CrossRef]

33. Guseman, E.H.; Eisenmann, J.C.; Laurson, K.R.; Cook, S.R.; Stratbucker, W. Calculating a continuous metabolic syndrome score using nationally representative reference values. Acad. Pediatr. 2018, 18, 589-592. [CrossRef] [PubMed]

34. Cook, S.; Auinger, P.; Huang, T.T.K. Growth curves for cardio-metabolic risk factors in children and adolescents. J. Pediatr. 2009, 155, S6.e15-S6.e26. [CrossRef] [PubMed]

35. Kang, G.D.; Guo, L.; Guo, Z.R.; Hu, X.S.; Wu, M.; Yang, H.T. Continuous metabolic syndrome risk score for predicting cardiovascular disease in the Chinese population. Asia. Pac. J. Clin. Nutr. 2012, 21, 88. [PubMed]

36. Berry, J.D.; Lloyd-Jones, D.M.; Garside, D.B.; Greenland, P. Framingham risk score and prediction of coronary heart disease death in young men. Am. Heart J. 2007, 154, 80-86. [CrossRef] [PubMed]

37. Hemann, B.A.; Bimson, W.F.; Taylor, A.J. The Framingham Risk Score: An appraisal of its benefits and limitations. Am. Heart Hosp. J. 2007, 5, 91-96. [CrossRef]

38. Khanna, R.; Kapoor, A.; Kumar, S.; Tewari, S.; Garg, N.; Goel, P.K. Metabolic syndrome and Framingham risk score: Observations from a coronary angiographic study in Indian patients. Indian J. Med. Res. 2013, 137, 295.

(C) 2020 by the author. Licensee MDPI, Basel, Switzerland. This article is an open access article distributed under the terms and conditions of the Creative Commons Attribution (CC BY) license (http://creativecommons.org/licenses/by/4.0/). 